\title{
Note on porous rotating disk flow
}

\author{
N. Kelson* A. Desseaux ${ }^{\dagger}$
}

(Received 7 August 2000)

\begin{abstract}
We revisit the classical Kármán rotating disk problem. A series analysis is used to derive estimates of boundary conditions at the surface. Using these estimates, computed thermal and flow fields for large mass transfer through the disk are readily obtained using a shooting method. The relevance of the problem to practical flows is discussed briefly.
\end{abstract}

* Centre in Statistical Science and Industrial Mathematics, Queensland University of Technology, Qld 4001, AustraliA. mailto:n.kelson@fsc.qut.edu.au

$\dagger$ Institut Universitaire de Technologie, Valenciennes, FrancE.

${ }^{0}$ See http: //anziamj . austms .org. au/V42/CTAC99/Kels for this article and ancillary services, (c) Austral. Mathematical Soc. 2000. Published 27 Nov 2000. 


\section{Contents}

1 Introduction

C838

2 Governing equations

C839

3 Analysis for $W_{s}=0$

C841

4 Analysis for $W_{s} \neq 0$ and $\zeta$ small

C842

5 Numerical results

C846

6 Discussion and conclusion

C852

References

C853

\section{Introduction}

The problem of flow over a rotating disk has a long history, with von Kármán originally describing similarity transformations that enable the Navier-Stokes equations for an isothermal, impermeable rotating disk to be reduced to a system of coupled ordinary differential equations [10]. Subsequently, an extensive literature has built up on this and closely related flows, especially with regard to an apparent non-uniqueness of solutions of the governing equations. For a review see Zandbergen \& Dijkstra [12]. 
In this work we re-examine the flow of a porous heated rotating disk, motivated by the view that this problem can serve as a prototype for practical swirl flows. In combustors, for example, the swirl is often generated by a fan or rotating honeycomb so that the upstream flow is close to solid-body rotation with a nearly uniform axial velocity profile (see, e.g. [4]). A shooting method is used to compute solutions to the resulting two-point asymptotic boundary value problem, where estimates for the surface boundary conditions are needed to initiate the iterative process. The dependence of the computations on the latter is investigated, and for strong injection, we find that the success of the procedure depends very sensitively on these initial guesses. Thus, the purpose of this communication is to point out basic results relating to the flow and the numerical scheme. In the following, a series solution is developed for mass transfer through the disk, and is compared with existing analyses to obtain approximate boundary conditions for high suction or injection. Thereafter, sample computations using the derived boundary conditions are presented to demonstrate their efficacy, particularly for high injection rates. Finally, the relevance of the results to practical flows is discussed briefly.

\section{Governing equations}

Using cylindrical coordinates $(r, \phi, z)$, the disk rotates with angular velocity $\Omega$ about the $z$-axis, and is at temperature $\theta_{0}$ compared with the ambient 
$\theta_{\infty}$. Following von Kármán, a dimensionless normal distance from the disk, $\zeta=z(\Omega / \nu)^{1 / 2}$ is introduced, along with the following representations for the radial, tangential and axial velocities:

$$
u=-\frac{1}{2} \Omega r W^{\prime}(\zeta), \quad v=\Omega r V(\zeta), \quad w=\sqrt{\Omega \nu} W(\zeta)
$$

The Navier-Stokes equations reduce in this case to the coupled system

$$
2 W^{\prime \prime \prime}-2 W^{\prime \prime} W+\left(W^{\prime}\right)^{2}-4 V^{2}=0, \quad V^{\prime \prime}-V^{\prime} W+V W^{\prime}=0
$$

The dimensionless radial velocity can be found, as needed, via the equation $U=-W^{\prime} / 2$. Further, by introducing the dimensionless variable $T(\zeta)=$ $\left(\theta-\theta_{\infty}\right) /\left(\theta_{0}-\theta_{\infty}\right)$, the temperature distribution may be computed after the velocity field via the reduced equation

$$
T^{\prime \prime}=\operatorname{Pr} W T^{\prime}
$$

where $\operatorname{Pr}$ is the Prandtl number.

For uniform suction $\left(W_{s}<0\right)$ or injection $\left(W_{s}>0\right)$ at the surface, the appropriate boundary conditions are

$$
\begin{array}{rlll}
\zeta=0: & V=T=1, & U=0, & W=W_{s} \\
\zeta \rightarrow \infty: & V=T=0, & U=0 &
\end{array}
$$

For computational purposes, the asymptotic boundary conditions are implemented on a finite domain of cutoff length $\zeta_{c}$. 


\section{Analysis for $W_{s}=0$}

Cochran [2] obtained an early approximate solution to the coupled system for the case of no suction or blowing $\left(W_{s}=0\right)$ via the matching of a power series near $\zeta=0$ with an asymptotic series valid for $\zeta$ large. The leading terms of the power series solution to (2) and (4a) valid near $\zeta=0$ are

$$
\begin{aligned}
V & =1+b \zeta-\frac{a}{3} \zeta^{3}-\frac{1+a b}{12} \zeta^{4}-\cdots \\
W & =a \zeta^{2}+\frac{1}{3} \zeta^{3}+\frac{b}{6} \zeta^{4}+\cdots
\end{aligned}
$$

where $a$ and $b$ are constants.

For $\zeta$ large, formal expansions of the solution of (2) and (4b) can be found in powers of $e^{-c \zeta}$, where $c$ is a constant. The latter describe flow drawn in axially from infinity, consistent with the rotating disk acting as a kind of centrifugal fan. The leading terms are

$$
\begin{aligned}
V & =B e^{-c \zeta}-\frac{B\left(A^{2}+B^{2}\right)}{12 c^{4}} e^{-3 c \zeta}+\cdots \\
W & =-c+\frac{2 A}{c} e^{-c \zeta}-\frac{A^{2}+B^{2}}{2 c^{3}} e^{-2 c \zeta}+\frac{A\left(A^{2}+B^{2}\right)}{6 c^{5}} e^{-3 c \zeta}+\cdots
\end{aligned}
$$

where $A$ and $B$ are constants. The values (as determined by Cochran) are

$$
a=-0.510, \quad b=-0.616, \quad c=0.886, \quad A=0.934, \quad B=1.208 .
$$




\section{Analysis for $W_{s} \neq 0$ and $\zeta$ small}

To find estimates for the surface boundary conditions when $W_{s} \neq 0$, the series for $V$ and $W$ given in (5) can be modified to include suction or injection. We begin by assuming the following expansions, which satisfy the boundary conditions at $\zeta=0$, namely

$$
V=1+\sum_{n=1}^{\infty} b_{n} \zeta^{n}, \quad W=W_{s}+\sum_{n=2}^{\infty} a_{n} \zeta^{n}
$$

where $a_{n}$ and $b_{n}$ are constants. Note that $a_{1}=0$ is required for the no-slip condition at the disk. The series (8) can be substituted into (2) to obtain the following. For brevity, only the first few terms are given subsequently.

$$
\begin{aligned}
V=1 & +b_{1} \zeta+\frac{b_{1} W_{s}}{2} \zeta^{2}+\frac{2-6 a_{3}+b_{1} W_{s}^{3}}{6 W_{s}} \zeta^{3} \\
& +\frac{2 W_{s}-12 a_{3} W_{s}+2 b_{1}+b_{1} W_{s}^{4}-6 a_{3} b_{1}}{24 W_{s}} \zeta^{4}+\cdots \\
W=W_{s} & +\frac{-1+3 a_{3}}{W_{s}} \zeta^{2}+a_{3} \zeta^{3}+\frac{3 a_{3} W_{s}+2 b_{1}}{12} \zeta^{4}+\cdots
\end{aligned}
$$

Now there is a singularity at $W_{s}=0$ in the above. To remove this we assume that the preceding series for $V$ and $W$ are bounded for both small $\zeta$ and for arbitrary amounts of suction or blowing through the surface. In particular, we require them to yield the corresponding series solution for 
$W_{s}=0$, equation (5), in the limit $W_{s} \rightarrow 0$. With these points in mind we consider (9) in the limit of no suction or injection. By a term by term comparison with (5), it follows that

$$
\lim _{W_{s} \rightarrow 0} \frac{-1+3 a_{3}}{W_{s}}=a, \quad \lim _{W_{s} \rightarrow 0} a_{3}=\frac{1}{3}, \quad \lim _{W_{s} \rightarrow 0} \frac{3 a_{3} W_{s}+2 b_{1}}{12}=\frac{b}{6}, \quad \ldots
$$

A solution to the above is

$$
a_{3}=\frac{1+a W_{s}}{3}, \quad b_{1}=b
$$

and substituting into (9), the following expressions for the tangential and axial velocities are obtained which are valid for $\zeta$ small:

$$
\begin{aligned}
& V=1+b \zeta+\frac{b W_{s}}{2} \zeta^{2}+\frac{-2 a+b W_{s}^{2}}{6} \zeta^{3}+\frac{-2-2 a b-4 a W_{s}+b W_{s}^{3}}{24} \zeta^{4}+\cdots \\
& W=W_{s}+a \zeta^{2}+\frac{1+a W_{s}}{3} \zeta^{3}+\frac{2 b+W_{s}+a W_{s}^{2}}{12} \zeta^{4}+\cdots
\end{aligned}
$$

For $W_{s}=0,(12)$ is identical to (5), so the above expressions represent the more general form of (5) to include suction or injection. Further, when $\left|W_{s}\right|$ is large, it is possible to get approximate closed form expressions for (12) by retaining only the highest term in each coefficient. In this case, we obtain

$$
\begin{aligned}
V & \approx 1+\frac{b}{W_{s}}\left(e^{W_{s} \zeta}-1\right) \\
W & \approx W_{s}+\frac{2 a}{W_{s}^{2}}\left(e^{W_{s} \zeta}-1-W_{s} \zeta\right)
\end{aligned}
$$


Equation (13) suggests that $W_{s} \zeta$ rather than $\zeta$ alone is a more suitable independent variable for the problem with large mass transfer through the disk. This observation suggests why in the work of Stuart [9], the ad-hoc choice of scaling variable $\eta=-W_{s} \zeta$ lead to a successful series expansion analysis valid for $\zeta$ small and strong suction. After rewriting the governing equations in terms of the new independent variable $\eta$, series solutions in descending powers of $W_{s}$ were sought, of the form

$$
V=\sum_{r=0}^{\infty}\left(-W_{s}\right)^{-r} V_{r}(\eta), \quad W=W_{s}+\sum_{r=0}^{\infty}\left(-W_{s}\right)^{-r} W_{r}(\eta)
$$

where $W_{r}$ and $V_{r}$ are functions to be determined. The above is substituted into the governing equations and successive powers of $W_{s}$ are equated to zero. A set of equations is obtained for the functions $W_{r}$ and $V_{r}$ that can be solved for with the appropriate boundary conditions at $\eta=0$ and $\infty$. The leading terms are

$$
\begin{aligned}
& V=e^{-\eta}+\frac{1}{12 W_{s}^{4}}\left(e^{-\eta}-6 \eta e^{-\eta}-e^{-3 \eta}\right)+O\left(W_{s}^{-8}\right) \\
& W=W_{s}+\frac{1}{2 W_{s}^{3}}\left(1-2 e^{-\eta}+e^{-2 \eta}\right)+O\left(W_{s}^{-7}\right)
\end{aligned}
$$

Estimates for $a$ and $b$, which respectively correspond to the conditions $W^{\prime \prime}(0) / 2$ and $V^{\prime}(0)$ at the disk, can now be obtained by comparing Taylor series expansions of the leading terms of Stuart's series with the leading terms of (12). Note that to get a non-zero estimate for $a$, the $O\left(W_{s}^{-3}\right)$ term for $W$ 
must be included. On comparing the corresponding expansions with (12) we find that for strong suction the predicted values of the constants are

$$
a=\frac{1}{2 W_{s}}, \quad b=W_{s}
$$

These estimates were also given by Sparrow \& Gregg [8] using an argument based on the assumption that for strong suction, $W \approx W_{s}$ everywhere.

For mass injection, the above approach fails because the resulting equations for $W_{r}$ and $V_{r}$ do not have a solution for $W_{s}>0$. We instead consider the work of Kuiken [5], who argued that for strong injection, the viscous boundary layer would be increasingly blown away from the disk. To account for the expected behaviour, the scaled variables used were, in terms of the present non-dimensionalisation, $\eta=R^{\frac{1}{2}} \zeta, f(\eta)=R^{\frac{1}{2}} W(\zeta)$ and $g(\eta)=V(\zeta)$, where $R=1 / W_{s}^{2}$. Series solutions were constructed in ascending powers of $R$ for $f$ and $g$ (e.g. $f=f_{0}+R f_{1}+R^{2} f_{2}+\ldots$ ). To leading order, it was found that

$$
f_{0}=g_{0}=\cos ^{2}(\eta)=1-\eta^{2}+\frac{\eta^{4}}{3}+\cdots
$$

We can again expand Kuiken's result and compare it with the leading terms of (12) via a little algebraic manipulation, noting that the function $g_{1}$ (see [5]) must be included in the expansion for $g$ to obtain a non-zero estimate for $b$. We find that for strong injection, the values of the constants 
are

$$
a=-\frac{1}{W_{s}}, \quad b=-\frac{2}{W_{s}^{3}}
$$

The predicted estimates for the constants found here are in good agreement with the numerical results presented below.

\section{$5 \quad$ Numerical results}

To check our analysis, solutions to equations (2) and (4) were computed using a shooting method utilising a fourth order Runge-Kutta scheme with Newton iteration to search for the values of $V^{\prime}(0)$ and $W^{\prime \prime}(0)$ at the surface.

The two-point non-linear boundary value problem given by (2) and (4) is numerically equivalent to solving the non-linear system of equations

$$
\mathbf{F}(\mathbf{I})=\left[V\left(\zeta_{c} ; \mathbf{I}\right), W^{\prime}\left(\zeta_{c} ; \mathbf{I}\right)\right]=\mathbf{0}
$$

where $\mathbf{I}=\left[V^{\prime}(0), W^{\prime \prime}(0)\right]$ and $V(\zeta ; \mathbf{I}), W(\zeta ; \mathbf{I})$ are solutions to the initial value problem given by (2) and (4a). Equation (19) is solved subject to choosing $\zeta_{c}$ large enough so that $\left|V\left(\zeta_{c}\right)\right|<\epsilon$ and $\left|W(\zeta)-W\left(\zeta_{c}\right)\right|<\epsilon$ for all $\zeta>\zeta_{c}$ and a given $\epsilon>0$. To ensure that results with a prescribed accuracy of least five significant digits could be reported, a number of preliminary computations were performed in order to determine sufficiently small values for the stepsize and the cutoff parameter $\epsilon$. 
After solution of the velocity field, the temperature field was obtained by solution of (3) subject to the relevant boundary conditions in (4), also using a shooting method.

Key features of the solutions are given in Table 1. Also recorded in the table are our estimated values for $\left(\zeta_{c}\right)_{\min }$, the smallest domain length that could be used to obtain results with the prescribed accuracy.

For strong suction $\left(W_{s} \ll 0\right)$, the tabulated values of $\left(\zeta_{c}\right)_{\min }$ indicate that accurate numerical solutions can be obtained on very short domains. When computing these solutions, we also noted that the numerical scheme was very stable, in that a final converged solution could be obtained using relatively poor initial guesses for the surface boundary conditions required to initiate the iterative scheme.

Thus, while the tabulated values of $V^{\prime}(0)$ and $W^{\prime \prime}(0)$ for $W_{s}<-2$ are in excellent agreement with the estimated boundary conditions for strong suction given in (16), the latter estimates are not really needed to obtain solutions for $W_{s}<-2$ using the present method.

For strong injection, however, the situation is reversed. We found that the success of the procedure for $W_{s}>0$ depends very sensitively on initiating the iterative scheme using increasingly accurate guesses for the boundary conditions $V^{\prime}(0)$ and $W^{\prime \prime}(0)$. In addition, larger values of $\zeta_{c}$ were needed to obtain solutions with the prescribed accuracy.

Thus, to successfully compute solutions for strong injection, the follow- 
TABLE 1: Characteristics of the solutions $(\operatorname{Pr}=0.71)$

\begin{tabular}{clllll}
\hline$W_{s}$ & $-W^{\prime \prime}(0) / 2$ & $-V^{\prime}(0)$ & $-T^{\prime}(0)$ & $-W\left(\zeta_{c}\right)$ & $\left(\zeta_{c}\right)_{\min }$ \\
\hline 8 & 0.124757 & $0.388547 \mathrm{e}-2$ & $0.215304 \mathrm{e}-17$ & 0.561486 & 40 \\
7 & 0.142387 & $0.577836 \mathrm{e}-2$ & $0.956098 \mathrm{e}-13$ & 0.579452 & 45 \\
6 & 0.165663 & $0.910681 \mathrm{e}-2$ & $0.139705 \mathrm{e}-9$ & 0.599445 & 40 \\
5 & 0.197566 & $0.154706 \mathrm{e}-1$ & $0.672820 \mathrm{e}-7$ & 0.621706 & 35 \\
4 & 0.243044 & $0.289211 \mathrm{e}-1$ & $0.107326 \mathrm{e}-4$ & 0.646457 & 30 \\
3 & 0.309147 & $0.602893 \mathrm{e}-1$ & $0.576744 \mathrm{e}-1$ & 0.674195 & 30 \\
2 & 0.398934 & 0.135952 & $0.110135 \mathrm{e}-1$ & 0.707811 & 25 \\
1 & 0.489481 & 0.302173 & $0.848848 \mathrm{e}-1$ & 0.760731 & 25 \\
0 & 0.510233 & 0.615922 & 0.325856 & 0.884477 & 20 \\
-1 & 0.389569 & 1.175222 & 0.793048 & 1.260561 & 13 \\
-2 & 0.242421 & 2.038527 & 1.437782 & 2.057726 & 8 \\
-3 & 0.165582 & 3.012142 & 2.135585 & 3.018209 & 6 \\
-4 & 0.124742 & 4.005180 & 2.842381 & 4.007771 & 5 \\
-5 & 0.999187 & 5.002661 & 3.551223 & 5.003992 & $<5$ \\
-10 & 0.050002 & 10.00033 & 7.100153 & 10.00050 & $<5$ \\
\hline
\end{tabular}




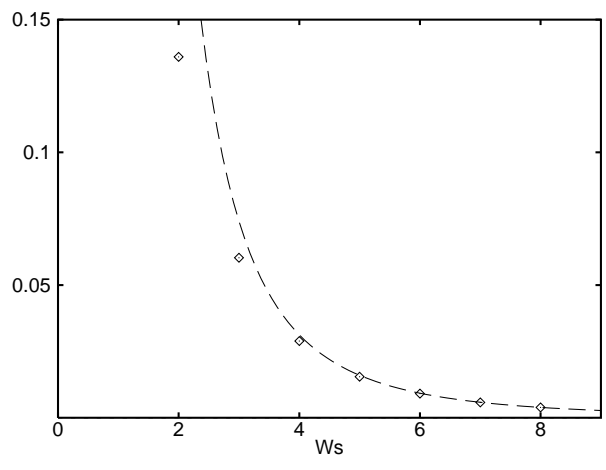

(a) $-V^{\prime}(0)$

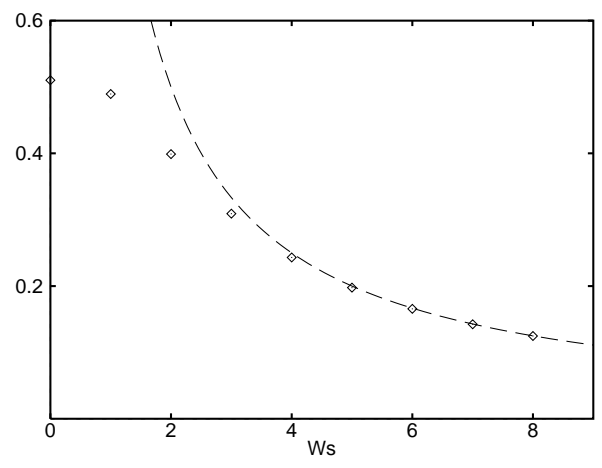

(b) $-W^{\prime \prime}(0) / 2$

Figure 1: Comparison of computed and estimated boundary conditions $V^{\prime}(0)$ and $W^{\prime \prime}(0)$ for $W_{s}>0$ : (a) Computed value of $-V^{\prime}(0)(\diamond)$ compared with $-b=1 / W_{s}^{3}(--)$; (b) Computed value of $-W^{\prime \prime}(0) / 2(\diamond)$ compared with $-a=1 / W_{s}(--)$.

ing procedure was adopted. Computations were started using the derived estimates for strong injection given in (18) on a small domain, and the computed values at the disk were used as initial guesses for computations using successively larger $\zeta_{c}$ until errors were reduced to the required levels.

A comparison of the computed and estimated boundary conditions $V^{\prime}(0)$ and $W^{\prime \prime}(0)$ for injection $\left(W_{s}>0\right)$ is illustrated in Figure 1. For higher injection rates, the derived estimates of the required boundary conditions given in (18) are seen to be in very good agreement with the corresponding 


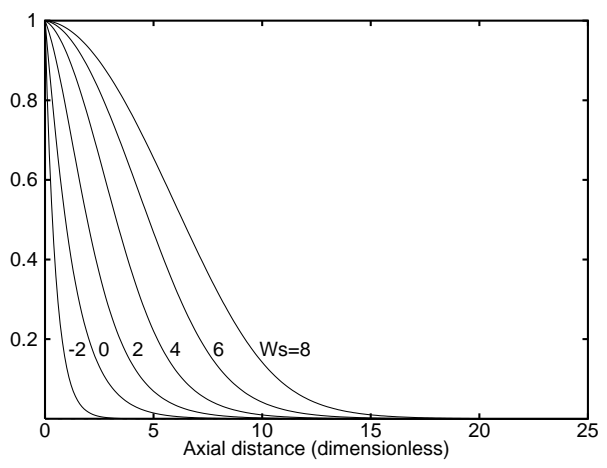

(c) Tangential velocity

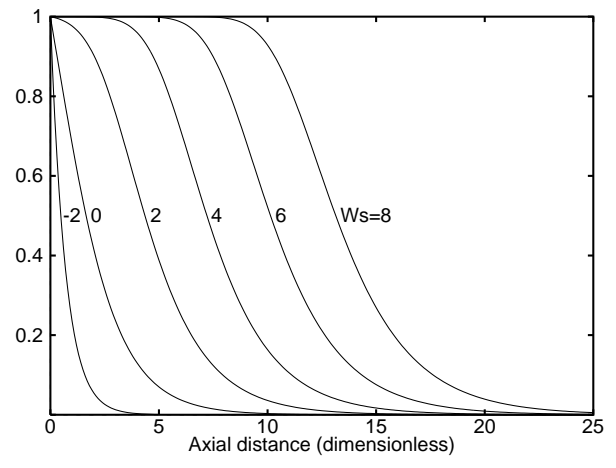

(d) Temperature

Figure 2: Velocity and Temperature profiles for different $W_{s}$.

computed values.

Finally, the computed velocity and temperature profiles for $\operatorname{Pr}=0.71$ (corresponding to air) and different rates of mass transfer through the disk are shown in Figure 2. For strong suction, the axial velocity is nearly constant, the radial velocity is very small, and the tangential velocity and temperature decay rapidly away from the surface. The case of strong suction is of less interest as a prototype for practical swirl flows, and we give only one representative profile for $W_{s}=-2$. However, a number of profiles for injection are given in Figure 2, where the boundary layer is increasingly blown away from the disk to form an interlayer between the injection and outer flow regions, and the tangential velocity and temperature decay more slowly 
away from the surface.

Although not described above, another class of solutions could be obtained using the present scheme, for all suction and injection rates considered here. The second class is due to the finite domain length, where the flow near $\zeta_{c}$ is of the form $W \sim-\left(\zeta-\zeta_{c}\right)^{2} / \zeta_{c}{ }^{2}$ and $V=0$. For the impermeable case, solutions of this type were discussed by Mellor et al. [7] in their study of two-disk flows where the second disk is stationary and located at $\zeta_{c}$. We do not report details here, other than to note that our computations show that this type of two-disk flow does in fact exist for a range of suction and injection rates through the rotating disk, and can be obtained on a range of domain lengths by using initial conditions that differ only slightly from those needed for the single disk solutions.

We also note that in this work, no attempt was made to find any so-called pathological inviscid hump solutions [11] that are known to exist for $W_{s}=0$, but do not appear to have been reported for non-zero mass transfer through the disk. This highly numerically stiff class of solutions are characterised by regions of large flow reversal outside the initial boundary layer, where the peak velocities greatly exceed those found in the boundary layer itself, and their physical realism is open to question. 


\section{Discussion and conclusion}

We have used here a series analysis to derive estimates of surface boundary conditions for a rotating disk flow. Using these estimates, computed thermal and flow fields for large mass transfer through the disk were readily obtained using a shooting method.

As a prototype, the porous rotating disk flow would appear relevant to aid understanding of certain practical flows such as axisymmetric flow in combustors, particularly where the upstream flow conditions are close to solid-body rotation with a nearly uniform axial velocity profile. In such flows, breakdown to a region of recirculation or flow reversal downstream of the concentrated vortex core flow may be present for moderate to large swirl. In particular, in confined pipe flow, a number of experimental studies (e.g. [1]) have observed that in some cases the recirculation zone is not highly localised, but rather has a long "tail" of weak reverse flow that persists far downstream of the front stagnation point, a phenomenon for which no explanation has apparently been proposed to date. However, the porous rotating disk flow with injection considered here has the right features of a stagnation point and an outer reverse axial flow, which suggests the following qualitative explanation. For pipe flow where the upstream conditions are due to a confined rotating disk of finite radius with injection and sufficiently high swirl, the flow may be expected to be geometrically similar over at least some of the pipe radius, and the features as obtained here will be evident, including weak backflow far downstream from the initial point of flow reversal. For 
this confined flow, mass conservation will necessitate that regions of forward axial flow will be present, most likely near the pipe generators. The resulting wall boundary layers will initially tend to grow and envelope the region of flow reversal, but as the axial velocity decays downstream, the boundary layer growth may be insufficient to impact upon the remaining geometrically similar region of reverse axial flow near the pipe axis.

While no one definitive cause is known for why enclosed axisymmetric vortex breakdown occurs (for a review of the main theories, see e.g. Leibovich [6]), the idea that locally similar flow may be responsible does not appear to have been advanced in previous studies, and the validity of this idea is currently being investigated [3].

\section{References}

[1] S. A. Ahmed. Three component velocity measurements of an isothermal confined swirling flow. Proc. Instn Mech. Engrs Part G, 211:113-122, 1997. C852

[2] W. G. Cochran. The flow due to a rotating disc. Proc. Camb. Phil. Soc., 30:365-375, 1934. C841

[3] N. A. Kelson and D. L. S. McElwain. Analysis of turbulent confined highly swirling flow. Submitted. C853 
[4] N. A. Kelson and D. L. S. McElwain. Computation of turbulent swirling quarl burner flow. In M. P. Schwarz, M. R. Davidson, A. K. Easton, P. J. Witt, and M. L. Sawley, editors, Proc. 2nd Int. Conf. on CFD in the Minerals and Process Industries, pages 491-496, Melbourne, Australia, Dec 1999. CSIRO. C839

[5] H. K. Kuiken. The effect of normal blowing on the flow near a rotating disk of infinite extent. J. Fluid Mech., 47:789-798, 1971. C845, C845

[6] S. Leibovich. Vortex stability and breakdown: Survey and extension. AIAA J., 22:1192-1206, 1984. C853

[7] G. L. Mellor, P. J. Chapple, and V. K. Stokes. On the flow between a rotating and a stationary disk. J. Fluid Mech., 31:95-112, 1968. C851

[8] E. M. Sparrow and J. L. Gregg. Mass transfer, flow, and heat transfer about a rotating disk. J. Heat Transfer, 82:294-302, 1960. C845

[9] J. T. Stuart. On the effects of uniform suction on the steady flow due to a rotating disk. Quart. J. Mech. Appl. Math., 7:446-457, 1954.

C844

[10] T. von Kármán. Über Laminare und Turbulente Reibung. Zeitschrift für angewandte Mathematik und Mechanik (ZAMM), 1:233-252, 1921. C838 
[11] P. J. Zandbergen. New solutions of the Kármán problem for rotating flows. In Lecture notes in mathematics; 771, pages 563-581, Berlin, 1980. Springer-Verlag. C 851

[12] P. J. Zandbergen and D. Dijkstra. Von Kármán swirling flows. Annu. Rev. Fluid Mech., 19:465-491, 1987. C838 